\title{
INTERVALOS DE REFERÊNCIA PARA ELEMENTOS MENORES E TRAÇO EM CABELO HUMANO PARA A POPULAÇÃO DA CIDADE DO RIO DE JANEIRO - BRASIL
}

Maria Tereza W.D. Carneiro, Carmem L. Porto da Silveira *, Norbert Miekeley e Lúcia M. de Carvalho Fortes

Depto. de Química, Pontifícia Universidade Católica do Rio de Janeiro, Rua Marquês de São Vicente, 225, 22453-900

Rio de Janeiro - RJ

Recebido em 29/11/00; aceito em 6/7/01

\begin{abstract}
REFERENCE INTERVALS FOR MINOR AND TRACE ELEMENTS IN HUMAN HAIR FOR THE POPULATION OF RIO DE JANEIRO CITY, BRAZIL. Inductively coupled plasma mass spectrometry (ICP-MS) has been used for the determination of twenty minor and trace elements in hair samples from an urban population group $(\mathrm{N}=1775)$, aiming at the establishment of reliable hair reference intervals. Statistical evaluation of the data with respect to age, sex and anatomic region was performed by multivariant analysis and according to recommendations of the International Federation of Clinical Chemistry (IFCC). The results show that mainly age and anatomic region (scalp or pubis) influence significantly the concentration of several elements. Comparison of the here calculated reference intervals with those previously published and used by clinical laboratories for this population showed larger discrepancies and the need for an urgent revision of these data.
\end{abstract}

Keywords: hair analysis; reference intervals; ICP-MS.

\section{INTRODUÇÃO}

Um balanço mineral apropriado no organismo humano é considerado fundamental para prevenir, assim como corrigir problemas de saúde. A análise de tecido capilar foi proposta e está sendo utilizada, freqüentemente, para verificar este equilíbrio mineral, pois o cabelo apresenta alguns atributos ideais como, ser facilmente coletado e estocado, além de permitir que elementos menores e traço possam ser determinados por uma variedade de técnicas analíticas, devido às maiores concentrações encontradas, quando comparado com outros tecidos ou fluidos do corpo ${ }^{1,2}$. Em artigo recente, Pozebon et $a l^{3}$ revisaram os diversos aspectos relacionados à análise de cabelo e do seu uso como monitor biológico em diferentes situações: verificação de consumo de drogas e de exposição ambiental; medicina de trabalho e medicina ortomolecular; criminalística; e outras.

Espécies químicas são incorporadas pelo cabelo através de diferentes fontes e mecanismos endógenos e exógenos ${ }^{4-6}$. As fontes endógenas são, definitivamente, as mais importantes quando se deseja fazer uma avaliação do estado de saúde de um indivíduo, no que diz respeito a anomalias fisiológicas, desequilíbrio nutricional ou intoxicação ambiental ${ }^{7-10}$. Muito embora as fontes exógenas contribuam, também, para a concentração total de elementos no cabelo, etapas de lavagem devem ser realizadas com a finalidade de minimizar problemas na interpretação biomédica dos resultados ${ }^{11,12}$.

$\mathrm{O}$ uso do cabelo para avaliação de desequilíbrios minerais (o chamado mineralograma) tem sido criticado, ultimamente, devido ao pouco embasamento científico disponível até agora e à propaganda desleal de alguns laboratórios clínicos, os quais realizam este exame em grande número e com alto lucro financeiro. Alem disso, estudos em que anomalias nos níveis de elementos menores e traço não se relacionam com estados fisiológicos modificados provocados por várias doenças, nem com a deficiência nutricional de um elemento em particular, e a falta de correlações existentes entre os teores de elementos no cabelo com os de outros indicadores biológicos

\footnotetext{
* e-mail: clportos@ rdc.puc-rio.br
}

(p.ex. sangue e urina), também contribui para que a utilidade do cabelo como monitor seja questionada ${ }^{13-15}$. Entretanto, a partir de um estudo amplo e envolvendo metodologias analíticas confiáveis, Miekeley et $a l .{ }^{16}$ e Fortes ${ }^{17}$, em publicações recentes, comprovaram que patologias endócrinas que afetam o equilíbrio ósseo refletem-se em excessos ou deficiências de elementos no cabelo (p.ex. Ca e P), sendo estas anomalias também observadas na urina. Pacientes, nos quais o desequilíbrio metabólico era causado pelo hiperparatireoidismo primário, voltaram a ter concentrações normais de elementos no cabelo e na urina, após extirpação cirúrgica parcial das glândulas paratireóide, mostrando a existência clara de correlações entre doenças e anomalias elementares no cabelo.

A dificuldade de se estabelecer os intervalos de referência (faixas de "normalidade") para elementos menores e traço, ou o uso de intervalos não adequados, são os principais problemas na utilização do cabelo como monitor biológico. Fatores como idade, sexo, dieta alimentar, local de residência, entre outros, podem contribuir, com maior ou menor influência, nas concentrações dos elementos no tecido capilar, provocando uma variabilidade na sua composição. Um outro aspecto, que também deve ser levado em conta, é a confiabilidade metrológica dos resultados analíticos produzidos e publicados. No cabelo, as concentrações de vários elementos essenciais (p.ex. Se, Mo, Cr, Co, V) e outros tóxicos (p.ex. As, Sb, Ag, $\mathrm{Hg}, \mathrm{Cd}, \mathrm{Pb}$ ) estão, freqüentemente, em níveis menores do que $\mu g / g$, ficando muito próximas ou abaixo do limite de quantificação da técnica de espectrometria de emissão atômica com fonte de plasma indutivamente acoplado (ICP-OES), utilizada na análise rotineira de cabelo pela maioria dos laboratórios clínicos, pelo menos até 1996. Baseado nos resultados de trabalho anterior do mesmo grupo $^{18} \mathrm{e}$ confirmados, recentemente, por Seidel et al. ${ }^{19}$, pode-se concluir que os intervalos de referência estabelecidos por importantes laboratórios clínicos internacionais, e citados como referência em artigos científicos e utilizados por médicos na interpretação do mineralograma, são, no mínimo, questionáveis.

O objetivo deste trabalho foi o estabelecimento de intervalos de referência de elementos menores e traço em cabelo para a população urbana da cidade do Rio de Janeiro, a partir de um considerável ban- 
co de dados, obtido pela aplicação da técnica de espectrometria de massas com fonte de plasma indutivamente acoplado (ICP-MS) e utilizando-se procedimentos metrologicamente validados. Pretendese, assim, suprir, no Brasil, a falta de dados confiáveis que possibilitem aos médicos e outros profissionais a utilização do cabelo como monitor biológico.

\section{MATERIAIS E MÉTODOS}

\section{Amostragem}

Amostras de cabelo de todos os indivíduos participantes foram coletadas por médicos ou profissionais da área, instruídos para seguir as normas recomendadas, internacionalmente, pelo Hair Analysis Standardization Board ${ }^{1}$. Isto é, com uma tesoura de aço inoxidável de alta qualidade (cirúrgica), retirar da região occipital, logo acima da nuca, uma mecha de cabelo pesando entre 250 e $500 \mathrm{mg}$. Apenas os três primeiros centímetros mais próximos ao escalpo eram utilizados. As amostras foram armazenadas em sacos de polietileno (5 $\mathrm{cm} \times 5 \mathrm{~cm}$ ), limpos e secos e identificadas por uma etiqueta onde constava o nome do indivíduo e data da coleta. Amostras da região pubiana foram coletadas pelos próprios pacientes, após rigorosas instruções e obedecendo os mesmos critérios anteriores. Uma ficha com informações sobre: local de residência; idade, sexo e raça; cor de cabelo e região anatômica da amostra coletada (escalpo ou púbis) foi obtida para cada indivíduo. Os critérios de inclusão atribuídos a cada participante do estudo foram: (1) ser morador da área urbana da cidade do Rio de Janeiro, (2) estar, aparentemente, clinicamente normal e (3) não ter feito tratamento cosmético no cabelo. Considerando a região do escalpo como preferencial, foram selecionados 1434 indivíduos para o grupo de referência, adicionalmente, foram selecionadas 341 amostras da região pubiana, totalizando 1775 indivíduos.

\section{Procedimentos analíticos}

A metodologia utilizada para a lavagem do cabelo, digestão ácida e posterior determinação dos elementos de interesse pela técnica de ICP-MS foi descrita por Fortes ${ }^{17}$. Resumidamente, o procedimento consiste na: (1) lavagem sucessiva do cabelo com acetona/ EXTRAN ${ }^{\circledR}$ $(1 \%$ v/v)/ água Milli-Q em banho de ultra-som, visando a eliminação dos elementos exógenos; (2) secagem à $60^{\circ} \mathrm{C}$ em estufa; (3) digestão de $200 \mathrm{mg}$ a $250 \mathrm{mg}$ da amostra com $2,5 \mathrm{~mL}$ de $\mathrm{HNO}_{3}$ (subbidestilado)e $1,0 \mathrm{~mL}$ de $\mathrm{H}_{2} \mathrm{O}_{2}$ (supra puro) em tubo de polipropileno rosqueado com tampa, em bloco digestor à $70{ }^{\circ} \mathrm{C}$; (4) diluição a volume final de $25 \mathrm{~mL}$; e (5) determinação dos elementos por ICP-MS.

\section{Espectrometria de massas com fonte de plasma indutivamente acoplado (ICP- MS)}

Foi utilizado o equipamento mod. ELAN 5000 da firma Perkin Elmer-Sciex. Para introdução da amostra foi empregada uma câmara de nebulização ciclônica (Glass Expansion, Camberwell, Austrália) em conjunto com um nebulizador concêntrico tipo Meinhard, operando com bomba peristáltica (mod. Minipulse, Gilson, França). Este sistema proporcionou maiores intensidades nos sinais analíticos (tipicamente $20-30 \%$ mais altas) e um menor tempo para rinsagem quando comparado com o sistema padrão do equipamento (nebulizador cross-flow com câmara de nebulização Ryton ${ }^{\circledR}$ ).

As condições operacionais relevantes do ICP-MS encontram-se resumidas na Tabela 1. Para otimização diária do desempenho do espectrômetro de massas e da fonte de plasma indutivamente
Tabela 1. Equipamento e parâmetros operacionais utilizados.

ESPECTROMETRIA DE MASSAS COM FONTE DE PLASMA INDUTIVAMENTE ACOPLADO (ICP-MS)

ELAN 5000 (PerkinElmer-Sciex)

Condições operacionais-padrão: Potência: $1050 \mathrm{~W}$; Vazão de Argônio (L/min): nebulizador - 0,950; plasma -15,00; auxiliar - 1,00.

Nebulizador: Meinhard com câmara ciclônica; taxa de aspiração: $1,0 \mathrm{~mL} / \mathrm{min}$

Amostrador/Skimmer: de platina

Sensibilidades (em s $\left.{ }^{-1}\right)$ : Mg-24 (15k); Rh-103 (70k-80k) e Pb-208 (87k) para uma solução de $10 \mu \mathrm{g}^{-1}$. L

Padrões Internos: ${ }^{45} \mathbf{S c},{ }^{69} \mathbf{G a},{ }^{115} \mathbf{I n},{ }^{205} \mathbf{T l}$. Cada padrão foi utilizado para corrigir as intensidades dos elementos/isótopos que o precedem na lista, p.ex. Sc-45 para corrigir as intensidades de Li-7 a Ca-44; Ga-69 para V-51 a Zn-66, etc.

Isótopos medidos (resolução normal e "peak hopping") : Li-7, Be9, B-11, C-13, Na-23, Mg-24, Al-27, P-31, S-34, Cl-35, K-39, Ca44, Sc-45, V-51, Cr-53, Mn-55, Fe-57, Co-59, Ni-60 (62), Cu-63 (65), Zn-66, Ga-69, Ge-72 (73), As-75, Br-79, Se-82, Sr-88, Mo98, Ag-107, Cd-111, In-115, Sn- 118, Sb-121, I-127, Ba-138, Au197, Hg-202, Tl-205, Pb-206 (207, 208), Bi-209, Th-232, U-238.

acoplado, foram feitos testes e ajustes iniciais, visando a obtenção de sinais analíticos altos associados à baixa formação de óxidos e íons bivalentes. Para esta finalidade, foi aspirada uma solução analítica mista contendo os elementos $\mathrm{Mg}, \mathrm{Rh}, \mathrm{Ce}, \mathrm{Ba}$ e $\mathrm{Pb}$ na concentração de $10 \mu \mathrm{g} . \mathrm{L}^{-1}$ de cada. Foram verificados, e se necessário reajustados, diariamente, os seguintes parâmetros para maximizar as intensidades dos íons $\mathrm{Me}^{+}$e para minimizar a formação de óxidos $(\mathrm{BaO}, \mathrm{CeO}):(1)$ alinhamento e distância tocha-amostrador e (2) vazão do gás nebulizador. Em condições operacionais otimizadas (vide Tabela 1), os resultados foram, tipicamente, os seguintes: sensibilidade para $\mathrm{Rh}(70 \mathrm{k}$ - $\left.80 \mathrm{k} \mathrm{s}^{-1}\right) ; \mathrm{Ba}^{+}(99,1 \%), \mathrm{BaO}(0,2 \%) ; \mathrm{Ba}^{2+}(0,7 \%) ; \mathrm{Ce}^{+}(97 \%)$ e $\mathrm{CeO}$ (3\%).

Para a determinação dos elementos foi utilizada a calibração externa quantitativa que consiste na confecção de curvas analíticas individuais para cada elemento. As mesmas foram produzidas, de maneira convencional, pela aspiração de soluções multielementares com concentrações conhecidas e variáveis dos analitos e registro das suas intensidades normalizadas em relação ao padrão interno. As soluções analíticas foram preparadas a partir dos padrões multielementares Merck-IV (Nr. 11355.0100, contendo os elementos: Ag, Al, B, Ba, $\mathrm{Ca}, \mathrm{Cd}, \mathrm{Co}, \mathrm{Cr}, \mathrm{Cu}, \mathrm{Fe}, \mathrm{Ga}, \mathrm{In}, \mathrm{K}, \mathrm{Li}, \mathrm{Mg}, \mathrm{Mn}, \mathrm{Na}, \mathrm{Ni}, \mathrm{Pb}, \mathrm{Sr}, \mathrm{Tl}$ e $\mathrm{Zn})$ e soluções monoelementares Titrisol ${ }^{\circledR}$, Merck (Hg, Se, P, Ge, $\mathrm{Be}, \mathrm{Mo}, \mathrm{Au}, \mathrm{Sb}, \mathrm{U}, \mathrm{Th}, \mathrm{Pd}, \mathrm{Br}, \mathrm{Ti}, \mathrm{Sn}, \mathrm{V}, \mathrm{Cl}, \mathrm{I}, \mathrm{S})$ por diluições adequadas com água Milli-Q (resistência > $16 \mathrm{M} \Omega$ ) e ajuste final da concentração de $\mathrm{HNO}_{3}$ para $0,5 \%$ (v/v) com $\mathrm{HNO}_{3}$ (bi-subdestilado). Elementos quimicamente incompatíveis com a solução de calibração acima mencionada, foram preparados em soluções separadas.

Para a correção de variações nas intensidades dos sinais analíticos, devido a efeitos tais como mudanças nas condições de excitação, transporte do analito e outros processos, foram usados $\mathrm{Sc}(\mathrm{m} / \mathrm{z}$ $=45), \mathrm{Ga}(\mathrm{m} / \mathrm{z}=69), \mathrm{In}(\mathrm{m} / \mathrm{z}=115)$ e $\mathrm{Tl}(\mathrm{m} / \mathrm{z}=205)$ como padrões internos. Cada padrão interno foi usado numa determinada faixa de massa para corrigir as intensidades dos elementos nela incluídos (Tabela 1). As concentrações (intensidades) dos padrões internos foram ajustadas de tal maneira que a presença natural destes elementos no cabelo não pudesse interferir na correção e foram mantidas, 
Tabela 2. Parâmetros de desempenho analítico obtidos na análise de cabelo por ICP-MS. Fator de diluição da amostra: 100 ( $0,25 \mathrm{~g}$ em $25 \mathrm{~mL})$. LD (limite de detecção); LQ (limite de quantificação).

\begin{tabular}{|c|c|c|c|c|c|}
\hline Isótopo & $\begin{array}{l}\text { Sensibilidade } \mathbf{S} \\
\quad\left(\mathrm{s}^{-1} \cdot \mu \mathrm{g}^{-1} \cdot \mathrm{L}\right)\end{array}$ & $\begin{array}{c}\text { Faixa de Calibração } \\
\left(\mathrm{mg} \cdot \mathrm{L}^{-1}\right)\end{array}$ & $\begin{array}{c}\text { Correlação } \\
\text { ( } \mathrm{r})\end{array}$ & $\begin{array}{l}\text { LD (3s) } \\
\left(\mu \mathrm{g} \cdot \mathrm{g}^{-1}\right)\end{array}$ & $\begin{array}{c}\text { LQ (10s) } \\
\left(\mu \mathrm{g} \cdot \mathrm{g}^{-1}\right)\end{array}$ \\
\hline \multicolumn{6}{|c|}{ ELEMENTOS TÓXICOS } \\
\hline $\mathrm{Ag}-107$ & 4593 & $0,00625-0,050$ & 0,9996 & 0,00040 & 0,0013 \\
\hline Al -27 & 4960 & $0,0312-0,250$ & 0,9997 & 0,013 & 0,043 \\
\hline As -75 & 562 & $0,00625-0,050$ & 0,9993 & 0,0015 & 0,0050 \\
\hline $\mathrm{Ba}-138$ & 10577 & $0,0312-0,250$ & 0,9998 & 0,010 & 0,034 \\
\hline $\mathrm{Cd}-111$ & 1044 & $0,00625-0,050$ & 0,9995 & 0,00039 & 0,0013 \\
\hline $\mathrm{Hg}-202$ & 1079 & $0,00625-0,050$ & 0,9999 & 0,0019 & 0,0064 \\
\hline $\mathrm{Ni}-62$ & 168 & $0,00625-0,050$ & 0,9986 & 0,0089 & 0,030 \\
\hline $\mathrm{Pb}-208$ & 7730 & $0,0312-0,250$ & 0,9997 & 0,0012 & 0,0040 \\
\hline $\mathrm{Sb}-121$ & 3679 & $0,0025-0,020$ & 0,9999 & 0,00054 & 0,0018 \\
\hline \multicolumn{6}{|c|}{ ELEMENTOS ESSENCIAIS } \\
\hline $\mathrm{Ca}-44$ & 261 & $1,256-10,05$ & 0,9996 & 1,3 & 4,4 \\
\hline Co - 59 & 5068 & $0,00625-0,050$ & 0,9988 & 0,00054 & 0,0018 \\
\hline $\mathrm{Cu}-65$ & 957 & $0,0687-0,550$ & 0,9975 & 0,0071 & 0,024 \\
\hline $\mathrm{Fe}-57$ & 127 & $0,0687-0,550$ & 0,9985 & 0,079 & 0,26 \\
\hline $\mathrm{Mg}-24$ & 1394 & $0,0687-0,550$ & 0,9999 & 0,028 & 0,093 \\
\hline $\mathrm{Mn}-55$ & 6296 & $0,00625-0,050$ & 0,9997 & 0,0041 & 0,014 \\
\hline Мo - 98 & 2570 & $0,0025-0,020$ & 0,9998 & 0,0012 & 0,0039 \\
\hline$P-31$ & 97 & $0,20-1,63$ & 1,0000 & 0,59 & 2,0 \\
\hline $\mathrm{V}-51$ & 4647 & $0,0025-0,020$ & 0,9999 & 0,00090 & 0,0030 \\
\hline $\mathrm{Zn}-66$ & 447 & $0,265-2,050$ & 0,9983 & 0,015 & 0,050 \\
\hline
\end{tabular}

rigorosamente, constantes em todos os tipos de amostras (brancos, padrões analíticos e soluções resultantes da decomposição das amostras de cabelo).

Nos elementos de maior interesse para os objetivos deste estudo, não se observaram interferências espectrais significativas causadas pela matriz do cabelo, desta forma, as suas correções através de equações elementares não foram consideradas necessárias, na maioria dos casos. Uma discussão detalhada sobre este assunto pode ser encontrada em Carneiro ${ }^{20}$.

As características de desempenho da metodologia foram determinadas de maneira usual (vide resultados). Para a validação da metodologia foi analisado um dos poucos materiais de referência certificado (MRC) com matriz de cabelo humano disponíveis no mercado internacional, o GBW-09101 do Shanghai Institute of Nuclear Research, China.

\section{Tratamento de dados}

As concentrações obtidas pela análise das amostras, juntamente com as respectivas informações cadastrais, eram enviadas, diretamente, para um arquivo de banco de dados, através de um programa de computador desenvolvido especialmente para este trabalho. A avaliação dos resultados em relação a idade, sexo e região anatômica de amostragem foi realizada através da análise de variância multivariada (two-way MANOVA, p-level: < 0,01), utilizando-se o pacote computacional Statística for Windows (vers. 5.1 da StatSoft Inc.). Para comparação das médias a posteriori foi aplicado o teste de Duncan. Observou-se que a distribuição de freqüência da concentração para todos os elementos foi desviada, assimetricamente, para a esquerda (Figura 1A), induzindo que estes dados apresentavam uma distribuição log-normal, como já foi relatado em outros estudos ${ }^{21}$. Após a transformação logarítmica dos valores de concentração, o critério “ $3 \mathrm{~s}$ ” foi, então, utilizado para eliminar os resultados discrepantes (outliers) ${ }^{22,23}$. Os intervalos de referência ( $1 \mathrm{~s}$ e $2 \mathrm{~s}$ ) foram calculados, posteriormente, com o auxílio de um outro programa desenvolvido para este trabalho, de acordo com os procedimentos indicados por Solberg 22,23 .

\section{RESULTADOS E DISCUSSÃO}

\section{Metodologia analítica}

A sensibilidade (S) e o limite de detecção (LD) das espécies em estudo foram determinados de forma usual, isto é, através da declividade das curvas analíticas ( $S=\operatorname{tg} \alpha=\mathrm{s}^{-1} \cdot \mu \mathrm{g}^{-1}$. L) e pela razão: $3 \mathrm{DP}_{\text {branco }} \times \mathrm{S}^{-1}$, onde DP corresponde ao desvio padrão de 10 medidas do branco da abertura. Adicionalmente, os limites de quantificação $\left(\mathrm{LQ}=10 \mathrm{DP}_{\text {branco }} \times \mathrm{S}^{-1}\right)$ foram calculados por apresentarem uma avaliação mais realista do poder de quantificação da metodologia. As curvas analíticas, obtidas a partir de quatro concentrações diferentes e do branco de calibração, apresentaram adequada linearidade ( $r$ > 0,998) para todos os elementos. Características de desempenho analítico, representativas para condições rotineiras de trabalho, encontram-se na Tabela 2.

Repetitividade e exatidão da metodologia desenvolvida foram avaliadas com auxílio do material de referência certificado, GBW09101. Foram feitas quatro decomposições independentes utilizando-se para cada uma delas, cerca de $150 \mathrm{mg}$ do material (pesados exatamente). As soluções resultantes foram analisadas por ICP-MS e para alguns elementos, adicionalmente, por ICP-OES. Os valores médios e os intervalos de confiança da média, segundo Student ${ }^{24}$, encontram-se na Tabela 3, junto com as concentrações referenciadas. Para a maioria dos elementos foram obtidas boas repetitividades, em geral, com um desvio entre as medidas $<5 \%$ para os elementos menores (p.ex. Ca, P, Zn, Mg) e $<10 \%$ para os elementos traço (p.ex. Se, Cr, Ni, Co, Al), resultando nos limites de confiança indicados na Tabela 3. Observou-se, dentro da incerteza do método utilizado e dos próprios valores de referência, uma boa concordância entre os resultados obtidos neste trabalho e os valores certificados. 
Para os elementos onde a técnica de ICP-OES apresentou sensibilidade suficiente, permitindo a sua determinação na mesma solução de decomposição da amostra, observou-se boa concordância entre as duas técnicas (ICP-MS e ICP-OES), validando-se desta forma as metodologias analíticas usadas ${ }^{17}$.

Tabela 3. Resultados obtidos pelas técnicas de ICP-MS e ICP-OES no material de referência certificado GBW-09101(cabelo humano) do Shanghai Institute of Nuclear Research, Academia Sinica, CHINA. Os valores, em $\mu \mathrm{g} . \mathrm{g}^{-1}$, correspondem à média de quatro decomposições independentes e os respectivos intervalos de confiança (IC) segundo Student ${ }^{24}$. Para os valores certificados é dado o desvio padrão (DP).

\begin{tabular}{cccc}
\hline Elemento & $\begin{array}{c}\text { Valor } \\
\text { Médio } \pm \text { IC } \\
\text { ICP-MS }\end{array}$ & $\begin{array}{c}\text { Valor } \\
\text { Médio } \pm \text { IC } \\
\text { ICP-OES }\end{array}$ & $\begin{array}{c}\text { Valor } \\
\text { Certificado } \\
(\mathrm{DP})\end{array}$ \\
\hline $\mathrm{Zn}$ & $185 \pm 6$ & $184 \pm 3$ & $189(8)$ \\
$\mathrm{Se}$ & $0,59 \pm 0,1$ & n.d & $0,58(0,05)$ \\
$\mathrm{Cr}$ & $4,64 \pm 0,38$ & $4,0 \pm 0,3$ & $4,77(0,38)$ \\
$\mathrm{Mg}$ & $97,4 \pm 1,5$ & $97 \pm 1$ & $105(6)$ \\
$\mathrm{As}$ & $0,54 \pm 0,02$ & n.d. & $0,59(0,07)$ \\
$\mathrm{Ca}$ & $1049 \pm 36$ & $1005 \pm 20$ & $1090(72)$ \\
$\mathrm{Fe}$ & $79 \pm 8$ & $71 \pm 0,5$ & $71,2(6)$ \\
$\mathrm{Mn}$ & $2,80 \pm 0,06$ & $2,72 \pm 0,15$ & $2,94(0,2)$ \\
$\mathrm{Cu}$ & $24,3 \pm 0,15$ & $23,7 \pm 0,13$ & $23(1,4)$ \\
$\mathrm{Sr}$ & $4,82 \pm 0,14$ & $4,45 \pm 0,03$ & $4,19(0,14)$ \\
$\mathrm{Hg}$ & $2,02 \pm 0,09$ & n.d. & $2,16(0,21)$ \\
$\mathrm{Pb}$ & $8,8 \pm 0,5$ & n.d. & $7,2(0,7)$ \\
$\mathrm{Ni}$ & $2,5 \pm 0,3$ & $2,3 \pm 0,1$ & $3,17(0,4)$ \\
$\mathrm{Cd}$ & $0,098 \pm 0,008$ & n.d. & $0,095(0,012)$ \\
$\mathrm{Al}$ & $12,2 \pm 1,4$ & $10,6 \pm 0,2$ & $13,3(2,3)$ \\
$\mathrm{Co}$ & $0,139 \pm 0,01$ & n.d. & $0,135(0,008)$ \\
\hline
\end{tabular}

Concentração de elementos menores e traço no cabelo da população do Rio de Janeiro e intervalos de referência

Antes de se iniciar qualquer estudo estatístico, deve-se estabelecer o tipo de distribuição de freqüência das variáveis estudadas. Gráficos de distribuição de freqüência em função da concentração dos elementos foram obtidos com este objetivo, para todos os elementos; exemplos encontram-se nas Figuras 1A a 1F. A transformação dos resultados de concentração em perfeita distribuição normal (Gaussiana), é ilustrada na Figuras 1B. Este comportamento foi observado para todos os elementos (exemplos podem ser vistos nas Figuras 1B a 1F) e está em concordância com resultados publicados por outros autores ${ }^{22,23}$.

A obtenção de intervalos de referência, a partir das distribuições de freqüência, está sujeita a uma série de procedimentos recomendados pelo Scientific Committee of the International Federation of Clinical Chemistry (IFCC) ${ }^{23,25,26}$. Dentre estes, está a partição da população de referência em termos de idade e sexo. A análise de variância multivariada (MANOVA) foi utilizada para avaliar o efeito do sexo e idade sobre os níveis de concentração dos elementos. A classificação dos participantes deste estudo em relação a estas duas variáveis é mostrada na Tabela 4. Somente foram incluídos neste estudo os elementos com concentrações médias no cabelo superiores a pelo menos cinco vezes o limite de quantificação (LQ) da metodologia analítica (vide as Tabelas 2 e 5). Amostras com concentrações de um elemento abaixo do limite de quantificação da metodologia analítica (LQ), perfazendo $<5 \%$ do número total de amostras do grupo de referência, foram eliminadas.
Tabela 4. Classificação dos indivíduos selecionados em termos de idade e sexo; cabelo do escalpo.

\begin{tabular}{cccc}
\hline \multirow{2}{*}{$\begin{array}{c}\text { IDADE } \\
\text { (anos) }\end{array}$} & \multicolumn{2}{c}{ SEXO } & TOTAL \\
\cline { 2 - 3 } $1-10$ & 23 & 15 & \\
$11-20$ & 40 & 48 & 38 \\
$21-30$ & 43 & 84 & 88 \\
$31-40$ & 76 & 209 & 338 \\
$41-50$ & 106 & 223 & 350 \\
$51-60$ & 73 & 132 & 224 \\
$61-70$ & 62 & 101 & 167 \\
$71-80$ & 39 & 46 & 93 \\
$>80$ & 4 & 10 & 14 \\
\hline TOTAL & 466 & 968 & 1434 \\
\hline
\end{tabular}

Tabela 5. Média das concentrações dos elementos menores e traço no cabelo do escalpo de acordo com o sexo: masculino $n=466$; feminino $n=968$. Os valores em negrito ( $\mathrm{Ag} \mathrm{e} \mathrm{Ni}$ ) apresentam diferença significativa $(\mathrm{p}<0,01)$.

\begin{tabular}{ccc}
\hline \multirow{2}{*}{ Elemento } & \multicolumn{2}{c}{ Concentração Média $(\mu \mathrm{g} / \mathrm{g})$} \\
\cline { 2 - 3 } & SEXO MASCULINO & SEXO FEMININO \\
\hline $\mathbf{A g}$ & $\mathbf{0 , 1 2}$ & $\mathbf{0 , 3 5}$ \\
$\mathrm{Al}$ & 7,90 & 8,76 \\
$\mathrm{As}$ & 0,08 & 0,07 \\
$\mathrm{Ba}$ & 1,62 & 2,18 \\
$\mathrm{Cd}$ & 0,17 & 0,17 \\
$\mathrm{Hg}$ & 1,80 & 1,28 \\
$\mathbf{N i}$ & $\mathbf{0 , 2 4}$ & $\mathbf{0 , 5 4}$ \\
$\mathrm{Pb}$ & 7,18 & 5,91 \\
$\mathrm{Sb}$ & 0,02 & 0,02 \\
\hline $\mathrm{Ca}$ & 361 & 411 \\
$\mathrm{Co}$ & 0,01 & 0,02 \\
$\mathrm{Cu}$ & 20,8 & 23,5 \\
$\mathrm{Fe}$ & 11,8 & 12,2 \\
$\mathrm{Mg}$ & 33,8 & 38,2 \\
$\mathrm{Mn}$ & 0,54 & 0,66 \\
$\mathrm{Mo}$ & 0,03 & 0,04 \\
$\mathrm{P}$ & 209 & 201 \\
$\mathrm{Sr}$ & 1,94 & 2,27 \\
$\mathrm{~V}$ & 0,02 & 0,03 \\
$\mathrm{Zn}$ & 187 & 190 \\
\hline
\end{tabular}

\section{Efeito do Sexo}

A Tabela 5 apresenta os valores médios de concentração, a mediana e o desvio padrão, de acordo com o sexo dos indivíduos, destacando-se em negrito aqueles que foram influenciados, significativamente $(p<0,01)$. Apenas Ag e Ni tiveram suas concentrações afetadas em função do sexo do indivíduo. Não pode ser excluída, entretanto, a possibilidade que as maiores concentrações de $\mathrm{Ni}$ e Ag em cabelo de mulheres representam uma contaminação exógena causado pela tintura de cabelo, não admitida por um número considerável de indivíduos, uma vez que esses elementos aparecem, frequientemente, como anomalias associadas a cabelos, confirmadamente tingidos, analisados e descartados deste estudo. 

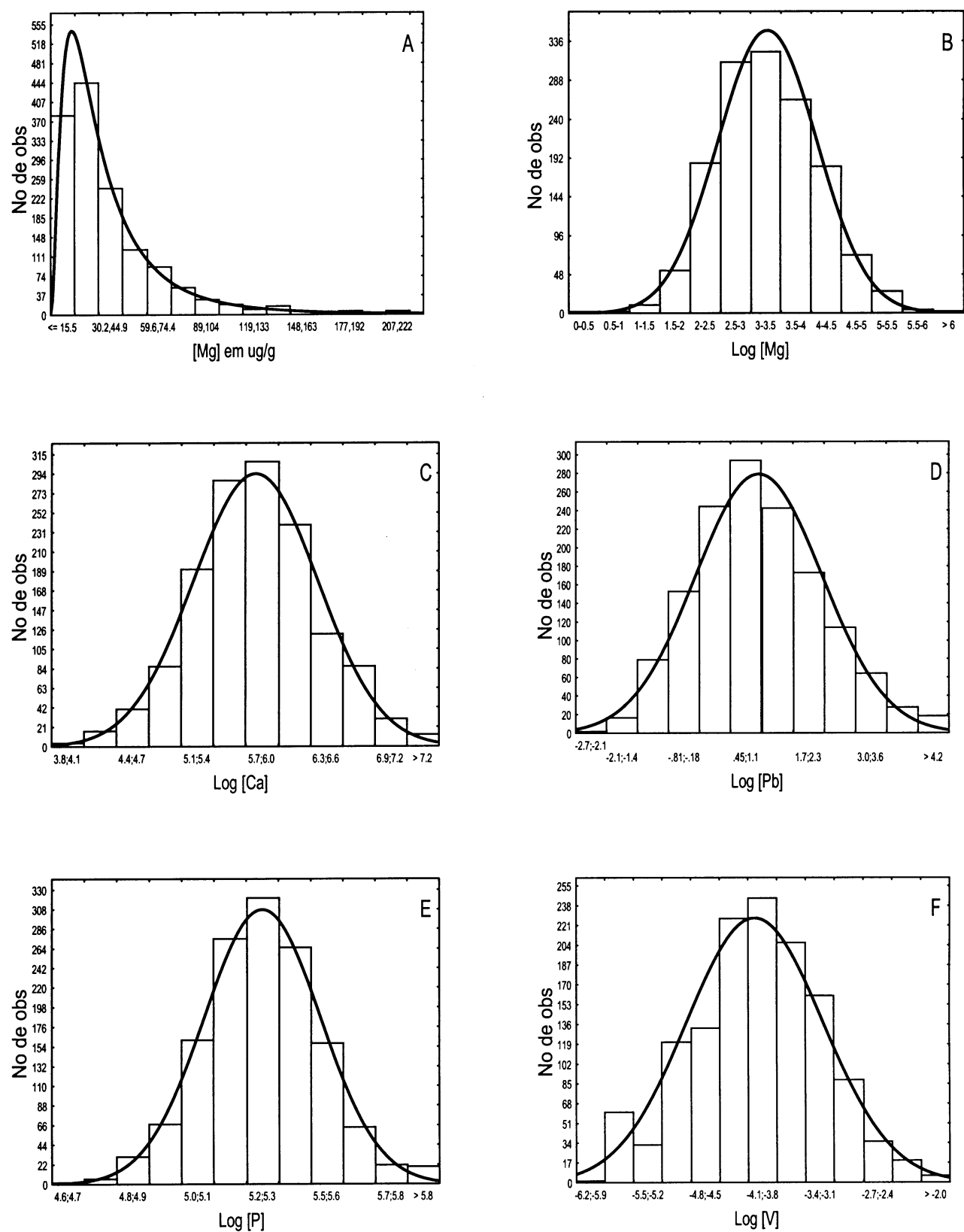

Figura 1. Exemplos de diagramas de freqüência (histogramas) das concentrações de diferentes elementos em cabelo (escalpo) da população mista. A figura $1 B$ mostra a log-transformação da distribuição assimétrica, Figura 1 A, em distribuição normal (Gaussiana).

\section{Efeito da Idade}

$\mathrm{O}$ fator idade afetou um número maior de elementos do que o sexo, indicando ser este efeito mais relevante quando pretende-se estabelecer intervalos de referência. As Figuras $2 \mathrm{~A}$ a $2 \mathrm{~F}$ mostram, para alguns elementos, a variação da concentração no cabelo com a idade dos indivíduos. $\mathrm{Al}, \mathrm{Cu}, \mathrm{Cd}$, Mo e $\mathrm{V}$ diminuem, de forma contínua, em função do aumento da idade (Figuras 2A, 2B e 2C), enquanto que outros elementos, tais como $\mathrm{Mg}, \mathrm{Sr}$ e $\mathrm{Ca}$, apresentam um máximo em torno de 40 anos (Figuras 2D, 2E e 2F). Considerando-se que $\mathrm{V}$, Mo e $\mathrm{Cu}$ são elementos essenciais, torna-se razoável imaginar que haja no organismo uma maior reserva relativa durante a infância, resultando, portanto, em uma maior concentração no cabelo na fase de desenvolvimento de um indivíduo. Já para Al, considerado tóxico ao organismo, uma avaliação mais cuidadosa deve ser realizada. $\mathrm{O}$ uso freqüente de piscinas na infância pode ser uma das causas dos teores mais elevados de $\mathrm{Al}$ encontrado para aquela faixa de idade, visto que este elemento é utilizado para tratamento de água e o procedimento de lavagem do cabelo não é capaz de remover, eficientemente, a contaminação exógena, nesta caso ${ }^{17}$. O mesmo efeito poderia ter influenciado os resultados obtidos para o $\mathrm{Cu}$.

Os dados encontrados para Cd estão de acordo com os publicados na literatura para áreas urbanas, geralmente mais contaminadas do que as áreas rurais ${ }^{2,20,27}$. De acordo com Paschal et al $^{28}$, crianças podem apresentar uma menor seletividade na absorção de alguns metais tóxicos, resultando em maior risco de contaminação na infância.

$\mathrm{O}$ comportamento seguido por $\mathrm{Mg}, \mathrm{Sr}$ e $\mathrm{Ca}$ em relação à idade foi muito semelhante. As Figuras 2D, 2E e 2F mostram que os teores destes elementos aumentam com a idade até os 40 anos, diminuindo a partir de então. Em virtude desses elementos apresentarem propriedades químicas semelhantes e desempenharem importante papel no desenvolvimento estrutural de um indivíduo ( $\mathrm{Ca}$ e $\mathrm{Mg}$ ), torna-se convincente o fato de exibirem idêntico comportamento em relação 

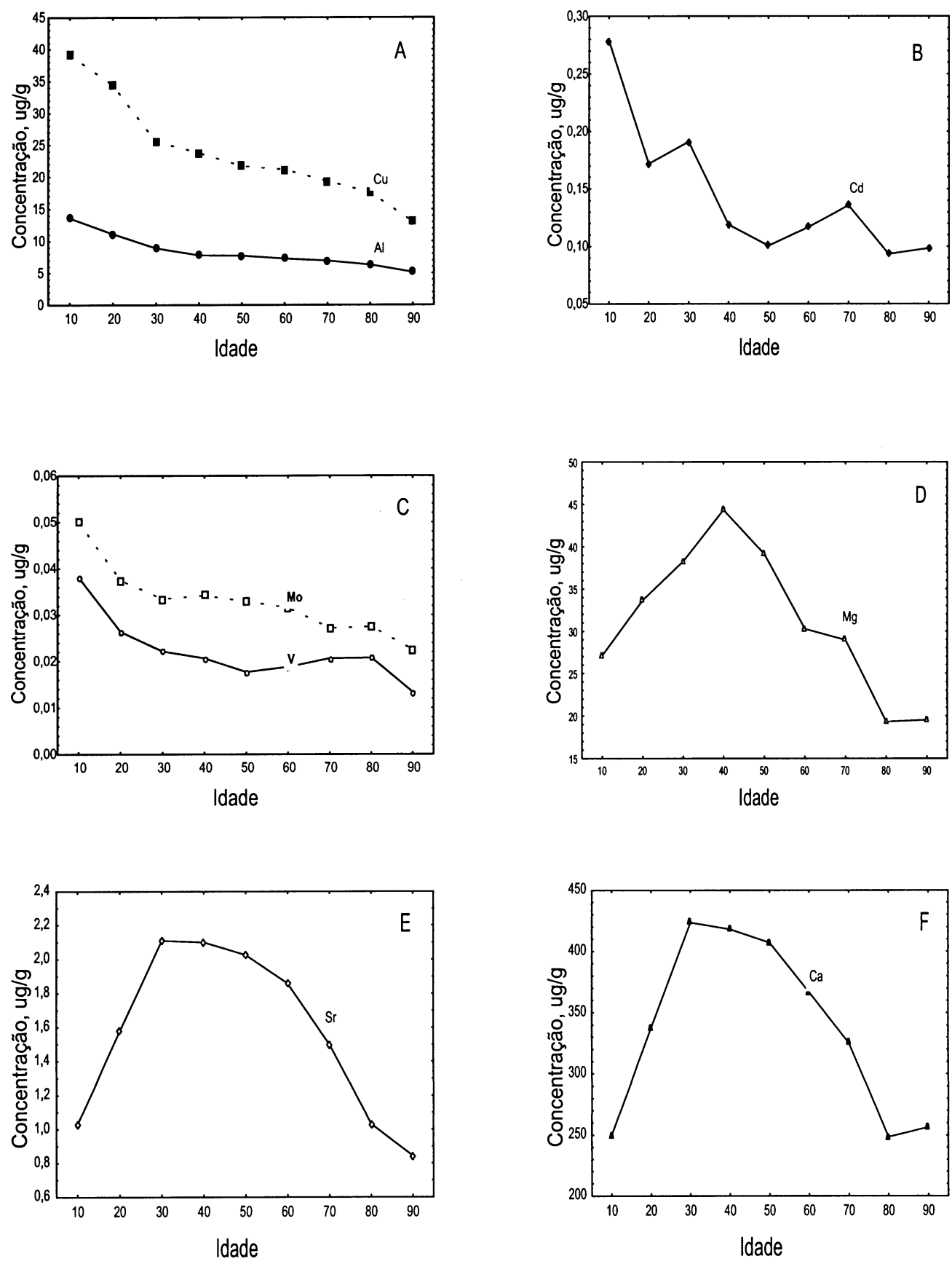

Figura 2. Variação da concentração média de alguns elementos no cabelo (escalpo) em função da idade.

à idade. Isto é ainda mais ressaltado quando se faz um estudo de correlação para estes elementos, como pode ser visto para $\mathrm{Ca}$ e $\mathrm{Mg}$ na Figura 3. A razão pela qual foram encontrados, na idade adulta, os maiores teores, deve estar relacionada ao fato do cabelo ser um tecido excretor ativo e refletir, desta forma, o equilíbrio existente no organismo humano para estes elementos . Na infância, estes elementos são conservados em maior extensão do que na idade adulta, resultando em teores menores, pois o desenvolvimento estrutural ainda não está completo ${ }^{29,30}$. Em artigo recente, Miekeley et al. ${ }^{16}$ mostraram que altas concentrações de $\mathrm{Ca}$ e $\mathrm{P}$ no cabelo, associadas ainda a outros elementos (p.ex. $\mathrm{Mg}, \mathrm{Ba}$ ), são freqüentes em mulheres na idade de pré-menopausa quando se iniciam desequilíbrios hormonais mais acentuados os quais aceleram a atividade osteoclástica e, desta forma, aumentam a transferência de elementos formadores do osso para os fluidos corporais e o cabelo.

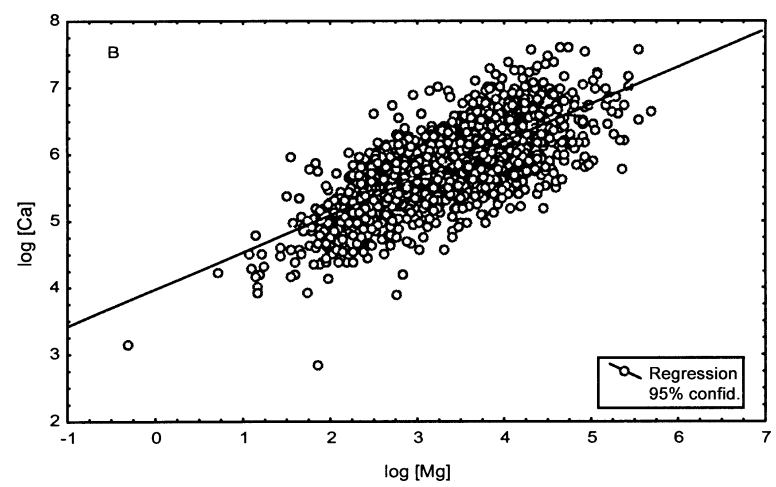

Figura 3. Correlação entre as concentrações de Ca e Mg em cabelo humano (escalpo; população mista; $N=1434 ; r=0,735$ ). 


\section{Efeito da Região Anatômica}

A região anatômica de onde o cabelo é amostrado, também é um fator importante que influi na concentração de elementos menores e traço. Foram analisadas amostras de cabelo pubiano $(n=341)$ numa tentativa inicial para se estabelecer intervalos de referência para este tipo de cabelo. As concentrações de As, Sb, Ba, P, Mg, Sr, Mn, Mo, $\mathrm{Ca}$ e $\mathrm{Co}$ foram, significativamente, diferentes quando o efeito da região anatômica foi testado através da análise de variância (multiway MANOVA, p-level: $<0,01)$. Todos esses elementos apresentaram concentrações maiores para a região pubiana quando comparado ao escalpo (Tabela 6).

Alguns outros autores também já investigaram a influência da região anatômica de onde a amostra de cabelo é coletada, sobre as concentrações de diversos elementos, entretanto, os resultados obtidos são discrepantes entre si e dos obtidos neste trabalho ${ }^{31-34}$. A diferença na velocidade de crescimento entre os dois tipos de cabelo pode ser uma das causas que justifique as divergências encontradas. O cabelo pubiano cresce a uma velocidade menor que o cabelo do escalpo (tipicamente: $0,2 \mathrm{~mm} / \mathrm{dia}$ X $0,4 \mathrm{~mm} / \mathrm{dia}$ ), o que torna o período de crescimento (fase anágena) mais longo, sugerindo maiores teores dos elementos na região pubiana ${ }^{2}$.

Por outro lado, a fase de repouso (telógena), durante a qual a fibra do cabelo é completamente expulsa do folículo, também é maior para o cabelo pubiano, quando comparado ao do escalpo. A razão crescimento/repouso é de $50 \%$, comparada aos $10 \%$ do escalpo, ou seja, a fase anágena tem a mesma duração da fase telógena, fazendo com que maiores concentrações sejam esperadas no escalpo ${ }^{2}$. Uma outra questão, que também deve ser levada em consideração, diz respeito às glândulas apócrinas que estão presentes na região pubiana e ausentes no escalpo. Essas glândulas são uma das fontes endógenas de incorporação de elementos menores e traço, contribuindo, desta forma, para a concentração dos elementos ${ }^{1,2}$. Além disso, não se pode

Tabela 6. Comparação entre as médias das concentrações de vários elementos menores e traço obtidas por ICP-MS em amostras de cabelo da região pubiana ( $n=341$ ) e do escalpo ( $n=1434$; população mista). Os valores em parêntesis correspondem aos desvios padrão. Elementos em negrito apresentam diferença significativa $(\mathrm{p}<0,01)$ entre os dois tipos de cabelo.

\begin{tabular}{|c|c|c|}
\hline \multirow[t]{2}{*}{ Elemento } & \multicolumn{2}{|c|}{$\begin{array}{l}\text { Valor Médio de Concentração } \\
\text { e (Desvio-Padrão) - } e m \mu g / g \text { - }\end{array}$} \\
\hline & Cabelo Pubiano & Cabelo do Escalpo \\
\hline $\mathrm{Pb}$ & $6,35(9,62)$ & $6,41(3,23)$ \\
\hline $\mathrm{Ag}$ & $0,18(0,30)$ & $0,26(0,61)$ \\
\hline As & $0,10(0,07)$ & $0,07(0,06)$ \\
\hline $\mathrm{Al}$ & $9,79(8,62)$ & $8,49(7,31)$ \\
\hline $\mathrm{Hg}$ & $1,20(1,41)$ & $1,44(1,84)$ \\
\hline Sb & $0,12(0,39)$ & $0,02(0,02)$ \\
\hline $\mathrm{Ni}$ & $0,39(0,32)$ & $0,41(0,55)$ \\
\hline $\mathrm{Cd}$ & $0,12(0,14)$ & $0,17(0,30)$ \\
\hline $\mathbf{B a}$ & $5,00(5,06)$ & $1,99(2,67)$ \\
\hline $\mathbf{P}$ & $539(375)$ & $204(46)$ \\
\hline Mg & $90,1(80,5)$ & $36,9(35,9)$ \\
\hline $\mathrm{Fe}$ & $14,8(7,3)$ & $12,1(5,7)$ \\
\hline $\mathrm{Zn}$ & $204(62)$ & $189(53)$ \\
\hline $\mathrm{Sr}$ & $5,58(5,63)$ & $2,17(2,65)$ \\
\hline $\mathrm{V}$ & $0,02(0,02)$ & $0,02(0,02)$ \\
\hline Mn & $1,81(2,31)$ & $0,62(0,76)$ \\
\hline Mo & $0,06(0,03)$ & $0,03(0,02)$ \\
\hline $\mathrm{Ca}$ & $980(884)$ & $395(268)$ \\
\hline $\mathrm{Cu}$ & $22,1(14,2)$ & $22,6(15,6)$ \\
\hline Co & $0,03(0,03)$ & $0,02(0,03)$ \\
\hline
\end{tabular}

esquecer que, assim como o cabelo do escalpo está sujeito à contaminação exógena, o cabelo de outras partes do corpo também sofre a contaminação de preparações cosméticas que contêm uma variedade de elementos traço em diferentes quantidades. Em razão do que foi exposto acima, um estudo mais detalhado sobre o cabelo da região pubiana deve ser realizado, para que seja possível uma avaliação mais apropriada do uso deste cabelo como bioindicador para elementos menores e traço no corpo humano.

\section{Intervalos de Referência}

Após terem sido avaliados os efeitos da idade, sexo e região anatômica, os intervalos de referência foram calculados para o cabelo do escalpo de acordo com os resultados obtidos. A população foi subclassificada em apenas duas faixas etárias, crianças e jovens com idades até 20 anos e adultos acima desta idade, levando-se em consideração o ainda pequeno universo amostral $(n=1434)$. Para alguns elementos verificou-se, entretanto, que, embora o teste estatístico tenha sido significativo, os intervalos de referência eram muito próximos, não sendo, portanto, necessária uma partição. Como alguns laboratórios clínicos comerciais apresentam os intervalos de referência utilizando $68 \%$ da fração central da distribuição de freqüência (1s), estes também foram calculados, junto com os obtidos utilizando-se 95\% da distribuição (2s), como é recomendado pela IFCC (Tabela 7). Quando compararam-se os resultados obtidos neste trabalho com os publicados por outros grupos de pesquisa ${ }^{35-39}$ e laboratórios clíni$\cos ^{18,19,40,41}$ (Tabela 8 ), constatou-se que para alguns elementos há

Tabela 7. Intervalos de referência (IR) para elementos menores e traço em cabelo do escalpo da população urbana do Rio de Janeiro. Para alguns elementos, os valores de IR foram calculados para duas faixa etárias: $<20$ anos e $>20$ anos.

\begin{tabular}{|c|c|c|c|c|c|}
\hline Elemento & $\begin{array}{l}\text { Idade } \\
\text { (anos) }\end{array}$ & Sexo & $\begin{array}{c}\text { Média } \\
(\mu g / g)\end{array}$ & $\begin{array}{c}\text { IR - 95\% } \\
(\mu g / g)\end{array}$ & $\begin{array}{c}\text { IR }-68 \% \\
(\mu g / g)\end{array}$ \\
\hline \multirow[t]{2}{*}{$\mathrm{Ag}$} & Todas & M & 0,12 & $<0,4$ & $<0,2$ \\
\hline & & $\mathrm{F}$ & 0,35 & $<2,2$ & $<0,5$ \\
\hline \multirow[t]{2}{*}{$\mathrm{Al}$} & $\leq 20$ & Todos & 13,33 & $<40$ & $<20$ \\
\hline & $>20$ & & 8,02 & $<27$ & $<12$ \\
\hline As & Todas & Todos & 0,07 & $<0,2$ & $<0,1$ \\
\hline $\mathrm{Ba}$ & Todas & Todos & 1,99 & $<9,1$ & $<3,3$ \\
\hline \multirow[t]{2}{*}{$\mathrm{Cd}$} & $\leq 20$ & Todos & 0,28 & $<1,5$ & $<0,45$ \\
\hline & $>20$ & & 0,16 & $<0,50$ & $<0,25$ \\
\hline $\mathrm{Hg}$ & Todas & Todos & 1,44 & $<6,4$ & $<2,4$ \\
\hline \multirow[t]{2}{*}{$\mathrm{Ni}$} & Todas & $\mathrm{M}$ & 0,24 & $<0,85$ & $<0,40$ \\
\hline & & $\mathrm{F}$ & 0,54 & $<2,65$ & $<0,90$ \\
\hline $\mathrm{Pb}$ & Todas & Todos & 6,41 & $<37$ & $<10$ \\
\hline $\mathrm{Sb}$ & Todas & Todos & 0,02 & $<0,049$ & $<0,032$ \\
\hline \multirow[t]{2}{*}{$\mathrm{Ca}$} & $\leq 20$ e $>50$ & Todos & 345 & $87-962$ & $158-524$ \\
\hline & $21-50$ & & 428 & $120-1117$ & $210-640$ \\
\hline $\mathrm{Co}$ & Todas & Todos & 0,02 & $0-0,056$ & $0,003-0,030$ \\
\hline \multirow[t]{2}{*}{$\mathrm{Cu}$} & $\leq 20$ & Todos & 73,48 & $4,6-358$ & $14-121$ \\
\hline & $>20$ & & 20,85 & $5,6-56$ & $10-31$ \\
\hline $\mathrm{Fe}$ & Todas & Todos & 12,10 & $4,5-27$ & $7,0-17$ \\
\hline \multirow[t]{2}{*}{$\mathrm{Mg}$} & $\leq 20$ e $>50$ & Todos & 31,34 & $4,5-114$ & $10-51$ \\
\hline & $21-50$ & & 41,62 & $6,3-148$ & $14-67$ \\
\hline Mn & Todas & Todos & 0,62 & $0,056-2,6$ & $0,15-1,0$ \\
\hline \multirow[t]{2}{*}{ Mo } & $\leq 20$ & Todos & 0,04 & $0,020-0,081$ & $0,028-0,057$ \\
\hline & $>20$ & & 0,03 & $0,012-0,076$ & $0,020-0,048$ \\
\hline $\mathrm{P}$ & Todas & Todos & 204,34 & $128-310$ & $160-249$ \\
\hline \multirow[t]{2}{*}{$\mathrm{Sr}$} & $\leq 20$ e $>50$ & Todos & 1,82 & $0,18-7,8$ & $0,45-3,0$ \\
\hline & $21-50$ & & 2,37 & $0,29-9,1$ & $0,69-3,9$ \\
\hline \multirow[t]{2}{*}{$\mathrm{V}$} & $\leq 20$ & Todos & 0,04 & $0,005-0,14$ & $0,011-0,059$ \\
\hline & $>20$ & & 0,02 & $0-0,062$ & $0,006-0,038$ \\
\hline $\mathrm{Zn}$ & Todas & Todos & 188,86 & $104-317$ & $138-240$ \\
\hline
\end{tabular}


Tabela 8. Intervalos de referência (IR - 68\%) para alguns elementos menores e traço em cabelo do escalpo, utilizados para a população urbana do Rio de Janeiro; fontes: Laboratórios Clínicos Internacionais (1994 e 1999) ${ }^{40,41}$. Para fins de comparação, dados da PUC-Rio foram, novamente, incluídos.

\begin{tabular}{|c|c|c|c|c|}
\hline \multirow[t]{2}{*}{ Elemento } & \multicolumn{3}{|c|}{ Laboratórios Clínicos } & \multirow[t]{2}{*}{ PUC-Rio } \\
\hline & B (1994) & A (1999) & A (1994) & \\
\hline \multicolumn{5}{|c|}{ Elementos Essenciais e outros } \\
\hline $\mathbf{L i}$ & $0,0010-0,50$ & $0,0010-0,53$ & $0,018-0,095$ & $<0,03 * *$ \\
\hline $\mathbf{N a}$ & $10-130$ & $10-130$ & $18-87$ & $\mathrm{n} / \mathrm{d}$ \\
\hline $\mathbf{K}$ & $5,0-40$ & $5,0-40$ & $8,8-38$ & $\mathrm{n} / \mathrm{d}$ \\
\hline $\mathbf{P}$ & $134-240$ & $134-240$ & $120-180$ & $160-250$ \\
\hline $\mathbf{B}$ & $0,080-4,0$ & $0,0080-4,0$ & $1,0-3,0$ & $<0,3 * *$ \\
\hline $\mathbf{C a}$ & $200-1600$ & $220-1600$ & $350-860$ & $180-600$ \\
\hline Mg & $20-130$ & $20-130$ & $40-110$ & $12-60$ \\
\hline $\mathbf{V}$ & $\mathbf{0 , 1 0 - 0 , 8 0}$ & $0,012-0,55$ & $\mathbf{0 , 3 5 - 0 , 8 0}$ & $0,006-0,04$ \\
\hline $\mathrm{Cr}$ & $0,20-1,2$ & $0,012-0,63$ & $0,78-1,0$ & $<0,32 * *$ \\
\hline $\mathbf{F e}$ & $4,2-23$ & $5,5-14$ & 6-15 & $7,0-17$ \\
\hline Mn & $0,20-1,8$ & $0,072-1,0$ & $0,26-0,75$ & $0,15-1,0$ \\
\hline $\mathbf{C u}$ & $13-59$ & $5,5-40$ & $13-35$ & $10-35$ \\
\hline Zn & $98-218$ & $142-248$ & $125-165$ & $138-240$ \\
\hline Mo & $0,50-15$ & $0,024-\mathbf{1 , 0}$ & $0,21-0,44$ & $0,02-0,05$ \\
\hline $\mathrm{Sr}$ & $0,29-5,4$ & $0,29-5,4$ & $1,0-7,6$ & $0,50-3,6$ \\
\hline Se & $2,5-15$ & $0,21-5,5$ & $0,38-0,70$ & $0,8-1,4$ \\
\hline Au & $0,002-\mathbf{0 , 7 5}$ & $0,002-\mathbf{0 , 7 5}$ & $\mathrm{n} / \mathrm{d}$ & $0,002-0,09$ \\
\hline $\mathbf{G e}$ & $0,004-\mathbf{0 , 4 0}$ & $0,004-\mathbf{0 , 4 3}$ & $0,20-1,6$ & $<0,12 * *$ \\
\hline Co & $0,050-1,0$ & $0,004-\mathbf{0 , 4 5}$ & $0,26-0,47$ & $0,003-0,03$ \\
\hline \multicolumn{5}{|c|}{ Elementos Tóxicos } \\
\hline $\mathbf{P b}$ & $<10$ & $<5,0$ & $<6,0$ & $<10$ \\
\hline Hg & $<1,2$ & $<1,3$ & $\mathrm{n} / \mathrm{d}$ & $<2,4$ \\
\hline Cd & $<0,8$ & $<0,75$ & $<1,0$ & $<0,27$ \\
\hline Ag & $\mathrm{n} / \mathrm{d}$ & $\mathrm{n} / \mathrm{d}$ & $<0,70$ & $<0,40$ \\
\hline $\mathbf{B a}$ & $<4,4$ & $<4,4$ & $0,30-3,5$ & $<3,0$ \\
\hline As & $<10$ & $<1,1$ & $<7,0$ & $<0,10$ \\
\hline $\mathbf{S b}$ & $\mathrm{n} / \mathrm{d}$ & $\mathrm{n} / \mathrm{d}$ & $<1,8$ & $<0,03$ \\
\hline Sn & $\mathrm{n} / \mathrm{d}$ & $\mathrm{n} / \mathrm{d}$ & $<3,0$ & $<0,34$ \\
\hline Al & $<18$ & $<17$ & $<12$ & $<14$ \\
\hline $\mathbf{N i}$ & $<2,2$ & $<1,1$ & $<1,6$ & $<0,70$ \\
\hline Th & $\mathrm{n} / \mathrm{d}$ & $\mathrm{n} / \mathrm{d}$ & $<8,5$ & $<0,004$ \\
\hline
\end{tabular}

**abaixo do limite de detecção; n.d = não determinado; os valores assinalados em negrito, são muito diferentes dos obtidos neste trabalho.

uma discrepância muito grande, podendo estar relacionada, entre outros, a fatores geoquímicos/ambientais e a dieta alimentar. Entretanto, há evidências que a pouca confiabilidade metrológica dos resultados analíticos utilizados para cálculo dos intervalos de referência parece ter uma contribuição grande, se não predominante, na disparidade dos resultados, como mostraram comparações interlaboratoriais realizadas por Miekeley et al. ${ }^{18} \mathrm{em} 1996$ e mais recentemente por Seidel et al. ${ }^{19}$, em 2001. A Tabela 8 compara os intervalos de referência (IR-68\%) de dois laboratórios clínicos (A e B) dos EUA com os resultados da PUC-Rio. Os dados indicados em negrito apresentam as maiores discrepâncias. Para alguns elementos traço (exemplos: B, V, Mo, Au, Ge, $\mathrm{Co}, \mathrm{As}, \mathrm{Th}$ ) foram reportados limites superiores de referência que ultrapassam em 100 a 2000 vezes (caso do Th) os sugeridos neste trabalho. Em 1999, o laboratório A corrigiu os seus intervalos superiores de referência para vários elementos ${ }^{41}$, incluindo o Mo (de $15 \mu \mathrm{g} . \mathrm{g}^{-1}$ para $1,0 \mu \mathrm{g} \cdot \mathrm{g}^{-1}$ ) e o As (de $10 \mu \mathrm{g} . \mathrm{g}^{-1}$ para $1,1 \mu \mathrm{g} . \mathrm{g}^{-1}$ ); outros elementos (B, V, Co, Au, Ge, Se), entretanto, estão ainda longe das suas concentrações verdadeiras no cabelo.

Resultados preliminares obtidos para outras regiões do Brasil (Minas Gerais e Goiânia) mostraram concentrações significativamente menores de vários elementos tóxicos $(\mathrm{Pb}, \mathrm{Cd}, \mathrm{Hg}, \mathrm{As}, \mathrm{Sb})$ no cabelo destas populações (predominantemente de origem rural), indicando, provavelmente, o efeito da maior poluição ambiental na cidade do Rio de Janeiro quando comparado aos ambientes rurais ${ }^{20}$.

\section{CONCLUSÕES}

Os resultados deste trabalho mostram que idade e região anatômica são os fatores predominantes quando se pretende estabelecer intervalos de referência para elementos menores e traço em cabelo humano. A influência do sexo na composição elementar do cabelo parece ser de menor relevância. Embora a literatura sugira o uso do cabelo pubiano como alternativa ao do escalpo, quando se deseja minimizar a contaminação exógena de elementos, os poucos resultados obtidos neste trabalho e os disponíveis na literatura mundial ainda não permitem o estabelecimento de intervalos de referência confiáveis. Uma comparação dos intervalos de referência obtidos para cabelo de escalpo neste trabalho, com os publicados por laboratórios clínicos internacionais, revela discrepâncias surpreendentes para vários elementos, recomendando uma revisão cuidadosa 
das metodologias analíticas utilizadas e cautela na utilização biomédica destes dados.

\section{AGRADECIMENTOS}

Os autores agradecem: ao apoio financeiro concedido pelo programa PADCT-II (FINEP, contrato $\mathrm{n}^{\circ}$ 65.92.0012); à CAPES e ao CNPq pelas bolsas recebidas (M.T.W.D. Carneiro e L.M.C. Fortes, respectivamente); a Leonardo R. Souza e Márcio Bonfim pelos programas de tratamento de dados; a ajuda exemplar do Álvaro J. Pereira na medição em ICP-MS e da Adriana dos Santos na preparação de centenas de amostras.

\section{REFERÊNCIAS}

1. Passwater, R. A.; Cranton, E. M.; Trace Elements, Hair Analysis and Nutrition, Keats Publishing Inc., New Canaan, CT, USA, 1983.

2. Chatt, A.; Katz, S. A.; Hair Analysis, Applications in the Biomedical and Environmental Sciences, VCH Publishers, New York, 1988.

3. Pozebon, D.; Dressler, V.L.; Curtius, A.J. ; Quim. Nova 1999, 22, 838.

4. Hopps, H.C.; Sci. Tot. Environ. 1977, 7, 71.

5. Chittlebrough, G. A.; Sci. Tot. Environ. 1980, 14, 53.

6. Peereboom, J.W. C.; Sci. Tot. Environ. 1985, 42, 1.

7. Chattopadhyay, A.; Jervis, R.E.; Trace-Subst. Environ. Health 1974, 8, 31.

8. Contiero, E.; Folin, M.; Biol. Trace Elem. Res. 1994, 40, 151

9. Ryan, D. E.; Hotzbecher, J.; Stuart, D.C.; Clin. Chem. 1978, 24, 1996.

10. Capel, I. D.; Pinnock, M.H.; Dorrell, H.M.; Williams, D.C.; Grant, C.G.; Clin. Chem. 1981, 27, 879.

11. Borella, P.; Rovesti, S.; Caselgrandi, E.; Bargellini, A.; Mikrochim. Acta 1996, 123, 271.

12. Ashraf, W.; Jaffer, M.; Mohammad, D.; Bull. Environ. Contam. Toxicol. 1994, 53, 516.

13. Hambidge,K.M.; Am. J. Clin. Nutr. 1982, 36, 943.

14. Riolin, R.S.; Am. J. Med. 1983, 75, 489.

15. Barret, S.; J. Am. Med. Assoc. 1985, 254, 1041

16. Miekeley, N.; Fortes, L. M. C; Porto da Silveira C. L.; Lima M. B.; J. Trace Elem. Med. Biol. 2001, 15, 46.

17. Fortes, L.M.C.; Tese de Doutorado, PUC-Rio, 1999, Rio de Janeiro, RJ.

18. Miekeley, N.; Carneiro, M.T.W.D.; Porto da Silveira, C.L.; Sci. Tot. Environ. 1998, 218, 9.
19. Seidel, S.; Kreutzer, R.; Smith, D.; McNeel, S.; Gilliss,D.; J. Am. Med. Assoc. 2001, 285, 67.

20. Carneiro, M.T.W.; Tese de Doutorado, PUC-Rio, 1999, Rio de Janeiro, RJ.

21. Poulsen, O.M.; Holst, E.; Christensen, J.M.; Pure Appl. Chem. 1997, 69, 1997.

22. Solberg, H. E.; In Reference Values in Laboratory Medicine; Gräsbeck, R.; Alström, T.; Ed.; John Wiley and Sons, Chichester, 1981, p 193.

23. Solberg, H.E.; Clin. Chim. Acta 1984, 137, 97F.

24. Miller, J. C.; Miller, J.N.; Statistics for Analytical Chemistry (2 $2^{\text {nd }}$. Ed.); Ellis Horwood; New York; 1992.

25. Solberg, H. E.; Clin. Chim. Acta 1987,165, 111

26. Dybkaer, R.; Clin. Chim. Acta 1983, 127, 441F

27. Wolfsperger, M.; Hauser, G.; Gössler, W.; Schlagenhaufen, C.; Sci. Tot. Environ. 1994, 156, 235.

28. Paschal, D. C.; DiPetro, E.S.; Phillips, D.L.; Gunter, E.W.; Environm. Res. 1989, 48, 17.

29. Bacso, J.; Uzonyi, I.; Katz, S.A.; Biol. Trace Elem. Res. 1987, 12, 383.

30. Moon, J.; Davison, A. J.; Smith, T.J.; Fadl, S.; Sci. Tot. Environ. 1988, $72,87$.

31. Wilhelm, M.; Ohnesorge, F.K.; Hötzel, D.; Sci. Tot. Environ. 1990, 92, 199.

32. DeAntonio, S.M.; Katz, S. A.; Scheiner, D.M.; Wood, J.D.; Clin. Chem. 1982, 28, 2411

33. Alder, J.F.; Samuel, A.J.; West, T.S.; Anal. Chim. Acta 1977, 92, 217.

34. Caroli, S.; Senofonte, O.; Violante, N.; Fornarelli, L.; Powar, A.; Microchem. J. 1992, 46, 171.

35. Senofonte, O.; Violante, N.; Fornarelli, L.; Beccaloni, E.; Powar, A.; Caroli, S.; Ann. Ist. Super. Sanità 1989, 25, 385.

36. Caroli, S.; Alimonti, A.; Femmine, P.D.; Petrucci, F.; Senofonte, O.; Violante, N.; Menditto, A.; Morisi, G.; Menotti, A.; Falconieri, P.; J. Anal. At.Spectrom. 1992, 7, 859.

37. Oluwole, A. F.; Ojo, J.O.; Durosinmi, M.A.; Asubiojo, O.I.; Akanle, O.A., Spyrou, N.M.; Filby, R.H.; Biol. Trace Elem. Res. 1994, 43, 443.

38. Kamakura, M.; Japan J. Hygiene 1983, 38, 823.

39. Caroli, S.; Alimonti, A.; Coni, E.; Petrucci, F.; Senofonte, O.; Violante, N.; Crit. Rev. Anal. Chem. 1994, 24, 363.

40. Op.cit. (Os intervalos de referência da Tabela 8, colunas A (1994) e B (1994), foram tirados de laudos do Doctors Data Inc. e do Trace Minerals Inc., ambos dos EUA).

41. Op.cit. (Os intervalos de referência da Tabela 8, coluna A (1999), foram tirados de laudo do Doctors Data Inc. em 1999 quando esse Laboratório reformulou seus valores) 